\title{
Neighbour-Sum-2-Distinguishing Edge-Weightings: Doubling the 1-2-3 Conjecture
}

\author{
Olivier Baudon $^{\mathrm{a}, \mathrm{b}}$, Julien Bensmail ${ }^{\mathrm{c}}$, Mohammed Senhaji ${ }^{\mathrm{a}, \mathrm{b}}$, Éric Sopena ${ }^{\mathrm{a}, \mathrm{b}}$ \\ ${ }^{a}$ Univ. Bordeaux, LaBRI, UMR5800, F-33400 Talence, France \\ ${ }^{b}$ CNRS, LaBRI, UMR5800, F-33400 Talence, France \\ ${ }^{c}$ Université Côte d'Azur, CNRS, Inria, I3S, France
}

\begin{abstract}
The 1-2-3 Conjecture asks whether every graph with no connected component isomorphic to $K_{2}$ can be 3-edge-weighted so that every two adjacent vertices $u$ and $v$ can be distinguished via the sum of their incident weights, that is the incident sums of $u$ and $v$ differ by at least 1 .

We here investigate the consequences on the 1-2-3 Conjecture of requiring a stronger distinction condition. Namely, we consider two adjacent vertices distinguished when their incident sums differ by at least 2 . As a guiding line, we conjecture that every graph with no connected component isomorphic to $K_{2}$ admits a 5 -edge-weighting permitting to distinguish the adjacent vertices in this stronger way.

We prove this conjecture for several classes of graphs, including bipartite graphs and cubic graphs. We then consider algorithmic aspects, and show that it is NP-complete to determine the smallest $k$ such that a given bipartite graph admits such a $k$-edge-weighting. In contrast, we show that the same problem can be solved in polynomial time for a given tree.
\end{abstract}

Keywords: 1-2-3 Conjecture; Difference-2 distinction; Bipartite graphs.

\section{Introduction}

Let $G$ be a graph, and $\omega$ be an edge-weighting of $G$. For every vertex $v$, one can compute its incident sum $\sigma_{\omega}(v)$ (or simply $\sigma(v)$ when no ambiguity is possible) of weights by $\omega$, being $\sigma(v):=\sum_{u \in N(v)} \omega(v u)$, where $N(v)$ denotes the set of neighbours of $v$. We call $\omega$ neighbour-sum-distinguishing if it yields a proper $\sigma$, i.e., we have $\sigma(u) \neq \sigma(v)$ for every edge $u v$ of $G$. It can be observed that every connected graph different from $K_{2}$ admits a neighbour-sum-distinguishing edge-weighting. Graphs with no connected component isomorphic to $K_{2}$ are thus said nice, with respect to neighbour-sum-distinguishing edgeweightings. For a nice graph $G$, it thus makes sense to investigate the smallest $k$ such that $G$ admits a neighbour-sum-distinguishing $k$-edge-weighting. This smallest $k$ is denoted by $\chi_{\Sigma}(G)$.

The 1-2-3 Conjecture, addressed by Karoński, Łuczak and Thomason [4], asks whether $\chi_{\Sigma}(G) \leq 3$ holds for every nice graph $G$.

1-2-3 Conjecture. For every nice graph $G$, we have $\chi_{\Sigma}(G) \leq 3$.

If true, the bound in the 1-2-3 Conjecture would be best possible, as attested for example by nice complete graphs and cycles with length not multiple of 4 . More generally,

\footnotetext{
"The second author was supported by ANR grant no. ANR-13-BS02-0007 "STINT" and PEPS grant POCODIS.
} 
it is NP-complete to decide whether $\chi_{\Sigma}(G) \leq 2$ holds for a given graph $G$, as first proved by Dudek and Wajc [1]. The same problem, however, can be handled in polynomial time when restricted to bipartite graphs, as recently shown by Thomassen, Wu and Zhang [7]. To date, the best result towards the 1-2-3 Conjecture is due to Kalkowski, Karoński and Pfender [3], who proved that $\chi_{\Sigma}(G) \leq 5$ holds for every nice graph $G$.

When designing neighbour-sum-distinguishing edge-weightings, the goal is to make adjacent vertices distinguishable via their incident sums. In ordinary neighbour-sumdistinguishing edge-weightings, adjacent vertices are considered distinguished as soon as their incident sums are distinct. We here investigate edge-weightings that permit to distinguish the adjacent vertices in a stronger way. Namely, we require adjacent vertices to have incident sums differing by at least 2 . An edge-weighting with this stronger requirement is said to be neighbour-sum-2-distinguishing throughout. As observed in upcoming Observation 2.1, a neighbour-sum-distinguishing $k$-edge-weighting can easily be turned into a neighbour-sum-2-distinguishing $2 k$-edge-weighting. Moreover, since $K_{2}$ does clearly not admit any neighbour-sum-2-distinguishing edge-weighting, the notion of nice graphs for neighbour-sum-distinguishing edge-weightings and for neighbour-sum-2-distinguishing edge-weightings coincide. Again, we can thus wonder about the smallest $k$ such that a given nice graph $G$ admits a neighbour-sum-2-distinguishing $k$-edge-weighting, which we denote by $\chi_{\Sigma>1}(G)$.

Our main goal in this paper is to study how $\chi_{\Sigma>1}$ behaves in general, in particular for graphs for which the parameter $\chi_{\Sigma}$ is well understood. As noted in upcoming Observation 2.1, the 1-2-3 Conjecture, if true, would imply that $\chi_{\Sigma>1}(G) \leq 6$ holds for every nice graph $G$. One could thus naturally wonder about a 1-2-3-4-5-6 Conjecture for neighboursum-2-distinguishing edge-weightings. It actually turns out that we did not manage to exhibit nice graphs $G$ with $\chi_{\Sigma>1}(G)=6$. On the other hand, we prove, throughout this paper, that for several common classes of nice graphs $G$ we have $\chi_{\Sigma>1}(G) \leq 5$. We are thus tempted to address the following.

Conjecture 1.1. For every nice graph $G$, we have $\chi_{\Sigma>1}(G) \leq 5$.

We here give first evidence towards Conjecture 1.1. We start in Section 2 by raising connections between neighbour-sum-distinguishing edge-weightings and neighbour-sum-2distinguishing edge-weightings, from which we deduce first bounds on $\chi_{\Sigma>1}$. In Section 3, we then prove Conjecture 1.1 for nice bipartite graphs, and investigate further some particular classes of bipartite graphs, from which we exhibit interesting aspects of the problem. The algorithmic aspects are considered in Section 4, where we first prove that it is NPcomplete to determine the exact value of $\chi_{\Sigma>1}$. This statement is showed to remain true even for bipartite graphs, which contrasts with the complexity of determining the exact value of $\chi_{\Sigma}$ for these graphs. We then show that determining the exact value of $\chi_{\Sigma>1}$ can be done in polynomial time for trees. Perspectives for future works are gathered in Section 5 .

\section{Preliminaries}

In this section, we point out general properties that actually apply to neighbour-sum$d$-distinguishing edge-weightings (for any $d \geq 1$ ), i.e., edge-weightings where the adjacent sums differ by at least $d$. For a given graph $G$, we denote by $\chi_{\Sigma>d-1}(G)$ the least integer $k$ such that $G$ admits a neighbour-sum- $d$-distinguishing $k$-edge-weighting. Note that the parameter $\chi_{\Sigma>0}$ actually corresponds to $\chi_{\Sigma}$. 
Obviously, by multiplying all weights assigned by a neighbour-sum-distinguishing $k$ edge-weighting by $d$, we obtain a neighbour-sum- $d$-distinguishing $d k$-edge-weighting.

Observation 2.1. For every nice graph $G$ and integer $d \geq 1$, we have $\chi_{\Sigma>d-1}(G) \leq$ $d \chi_{\Sigma}(G)$. In particular, $\chi_{\Sigma>1}(G) \leq 2 \chi_{\Sigma}(G)$.

Observation 2.1 already has several implications towards Conjecture 1.1. First, the 1-23 Conjecture, if true, would imply that $\chi_{\Sigma>1}(G) \leq 6$ holds for every nice graph $G$. Although we still do not know whether the 1-2-3 Conjecture is true, every partial result towards that conjecture can be adapted to Conjecture 1.1. In that line, perhaps the most interesting result to consider is the one due to Kalkowski, Karoński and Pfender [3], who proved that $\chi_{\Sigma}(G) \leq 5$ holds for every nice graph $G$. In our context, this and Observation 2.1 yield the following, which shows that, when requiring adjacent sums to differ by at least $d$, the number of needed weights grows linearly with $d$.

Corollary 2.2. For every nice graph $G$ and integer $d \geq 1$, we have $\chi_{\Sigma>d-1}(G) \leq 5 d$. In particular, $\chi_{\Sigma>1}(G) \leq 10$.

The bound in Corollary 2.2 is immediately improved for every graph $G$ for which we know that $\chi_{\Sigma}(G)<5$ holds. In particular, we have $\chi_{\Sigma>1}(G) \leq 6$ for every nice graph $G$ verifying the 1-2-3 Conjecture, which is very close to Conjecture 1.1. Let us recall, in particular, that the 1-2-3 Conjecture was verified for nice bipartite graphs, 3-chromatic graphs, nice complete graphs, and regular graphs with sufficiently large degree. We here refer the reader to the survey [6] by Seamone, wherein all such results are gathered.

As stated in Observation 2.1, by multiplying all weights assigned by a neighbour-sumdistinguishing $k$-edge-weighting by a same integer $d$, we get a neighbour-sum- $d$-distinguishing $d k$-edge-weighting since each $\sigma(v)$ is multiplied by $d$. This of course does not have to be true if one decreases (or increases) all weights by a same $d$, since, here, the effect on each $\sigma(v)$ depends on $d(v)$. There are situations, however, where this can be done safely.

Observation 2.3. Let $\omega$ be a neighbour-sum-distinguishing d-edge-weighting, $d \geq 1$, of a graph $G$ such that, for every edge uv (where $d(u) \geq d(v)$ ), we have $\sigma(u)<\sigma(v)$ (resp. $\sigma(u)>\sigma(v)$ ). Then, by decreasing (resp. increasing) all edge weights by a same integer $x$, we get another neighbour-sum-distinguishing d-edge-weighting of $G$.

Due to the fact that, in the context of Conjecture 1.1, we focus on edge-weightings assigning strictly positive weights, when decreasing edge weights we should also make sure that none becomes null or negative. Observation 2.3 can nevertheless be used when the smallest edge weight value assigned by $\omega$ is known. As an illustration, we improve Observation 2.1 for nice regular graphs.

Corollary 2.4. For every nice regular graph $G$ and integer $d \geq 1$, we have $\chi_{\Sigma>d-1}(G) \leq$ $d \chi_{\Sigma}(G)-(d-1)$. In particular, $\chi_{\Sigma>1}(G) \leq 2 \chi_{\Sigma}(G)-1$.

Corollary 2.4 notably implies that Conjecture 1.1 holds for nice complete graphs and 3-colourable regular graphs, as the 1-2-3 Conjecture was proved for such graphs. Other bounds also follow for regular graphs with larger degree, see [6].

\section{Conjecture 1.1 for bipartite graphs}

Since the 1-2-3 Conjecture holds for nice bipartite graphs (see [4]), from Observation 2.1 we directly get that $\chi_{\Sigma>1}(G) \leq 6$ holds for every nice bipartite graph $G$. It was actually 
proved by Thomassen, $\mathrm{Wu}$ and Zhang [7] that a bipartite graph $G$ satisfies $\chi_{\Sigma}(G)=3$ if and only if $G$ is an odd multicactus. Odd multicacti can be defined as follows. Start from a collection $C_{1}, \ldots, C_{m}$ of $m \geq 1$ cycles whose lengths are at least 6 and congruent to 2 modulo 4 , and colour the edges of the $C_{i}$ 's in a proper way using colours red and green. An odd multicactus is then any connected graph obtained by repeatedly applying the following operation: pick two connected components $G_{1}$ and $G_{2}$, and identify a green edge of $G_{1}$ with a green edge of $G_{2}$. Note that, in particular, every cycle whose length is congruent to 2 modulo 4 is an odd multicactus.

From this all, we directly get the following.

Corollary 3.1. For every nice bipartite graph $G$, we have

$$
\chi_{\Sigma>1}(G) \leq \begin{cases}4 & \text { if } G \text { is not an odd multicactus } \\ 6 & \text { otherwise. }\end{cases}
$$

In the next result, we improve Corollary 3.1 by completely proving Conjecture 1.1 for all nice bipartite graphs.

Theorem 3.2. For every nice bipartite graph $G$, we have $\chi_{\Sigma>1}(G) \leq 5$.

Proof. Let $A$ and $B$ denote the two partite sets of $G$. We prove a stronger statement, namely that $G$ admits a 5-edge-weighting such that the vertices from one of the partite sets have incident sum congruent to 2 or 3 modulo 5 , while the vertices from the other partite set have incident sum incident to 0 modulo 5. Such an edge-weighting is clearly neighbour-sum-2-distinguishing. In this setting, note that we can equivalently look for a neighbour-sum-2-distinguishing $\{0,1,2,3,4\}$-edge-weighting.

Assume the edges of $G$ are weighted in some way. In what follows, we repeatedly apply the following modification procedure for some given $\alpha \in\{1,2,3,4\}$. Let $u$ and $v$ be two distinct vertices from a same partite set, and let $P$ be a path (obviously of even length) from $u$ to $v$ in $G$. The procedure consists in modifying the weights of the edges of $P$ from one end to the other by alternatively subtracting and adding $\alpha$ (modulo 5 ), i.e., apply $-\alpha$, $+\alpha,-\alpha, \ldots,-\alpha,+\alpha$ to the weights along $P$. Note that this only alters the incident sums modulo 5 of $u$ and $v$, the two ends of $P$, which change by $-\alpha$ and $+\alpha$, respectively. Also, note that the "orientation" of $P$ is important, as the sum of its first vertex is altered by $-\alpha$ while the sum of its last vertex is altered by $+\alpha$. Hence, in what follows, though $G$ is not oriented, we consider paths from a vertex to another one, so that the first and last vertices are clearly identified.

We proceed as follows to obtain the claimed edge-weighting of $G$. Assume first that one of the two partite sets, say, $A$, has even size. Start from all edges being weighted 0 . Then repeatedly consider two new vertices $u$ and $v$ of $A$, and apply the modification procedure above with $\alpha=2$ onto a path from $u$ to $v$ in $G$. As stated, only the incident sums of $u$ and $v$ modulo 5 are altered, from 0 to 3 and 2, respectively. Once the process has been applied for all pairs of vertices, we get the desired neighbour-sum-2-distinguishing $\{0,1,2,3,4\}$-edge-weighting of $G$.

Assume now that $A$ and $B$ both have odd size. Repeating the exact same arguments as in the previous case on pairs of vertices of, say, $A$, we can deduce a $\{0,1,2,3,4\}$-edgeweighting of $G$ where all vertices of $A$ but one vertex $u$ have incident sum congruent to 2 or 3 modulo 5 , while all vertices of $B$ have incident sum congruent to 0 modulo 5 . In particular, also $u$ has incident sum congruent to 0 modulo 5 . If $A$ has two vertices $v_{1}, v_{2}$ with incident sum congruent to 2 (resp. 3) modulo 5 , then we consider one path $P_{1}$ from $u$ to $v_{1}$ and one path $P_{2}$ from $u$ to $v_{2}$, and apply the modification procedure for $\alpha=1$ (resp. 
$\alpha=4=-1$ modulo 5) onto $P_{1}$ and $P_{2}$. This affects the incident sums of $v_{1}$ and $v_{2}$ which are now congruent to 3 (resp. 2) modulo 5 , while $u$ now has incident sum congruent to 2 (resp. 3) modulo 5. The edge-weighting is thus as desired.

The only situation where the previous case does not apply is when $|A|=|B|=3$. In that case though, it can easily be checked by hand that $G$ always admits a neighbour-sum2-distinguishing $\{0,1,2,3,4\}$-edge-weighting.

Although Theorem 3.2 completely proves Conjecture 1.1 for nice bipartite graphs, in the rest of this section we study bipartite graphs further. Namely, we deduce the exact value of $\chi_{\Sigma>1}$ for nice paths and cycles, and prove a refinement of Conjecture 1.1 for odd multicacti. These results support some observations to be raised in concluding Section 5 .

In all upcoming proofs, we will deal with adjacent degree-2 vertices; for such a configuration, the following observation obviously applies.

Observation 3.3. Let $\omega$ be a neighbour-sum-2-distinguishing edge-weighting of a graph $G$, and $u v$ be an edge such that $d(u)=d(v)=2$. Assuming $u^{\prime}$ (resp. $v^{\prime}$ ) denotes the neighbour of $u\left(\right.$ resp. $\left.v^{\prime}\right)$ different from $v$ (resp. u), the weights $\omega\left(u^{\prime} u\right)$ and $\omega\left(v^{\prime} v^{\prime}\right)$ differ by at least 2 .

\subsection{Paths}

We denote by $P_{\ell}$ the path of length $\ell$. Therefore, the path $P_{1}=K_{2}$ is not nice. In the next result, we determine the value of $\chi_{\Sigma>1}\left(P_{\ell}\right)$ for every $\ell \geq 2$.

Theorem 3.4. For every path $P_{\ell}, \ell \geq 2$, we have

$$
\chi_{\Sigma>1}\left(P_{\ell}\right)= \begin{cases}2 & \text { if } \ell=2, \\ 3 & \text { if } \ell>2 \text { and } \ell \equiv 0,2,3 \quad(\bmod 4), \\ 4 & \text { otherwise }\end{cases}
$$

Proof. Recall that $\chi_{\Sigma>1}\left(P_{\ell}\right) \leq 4$ holds for every $\ell \geq 2$, by Corollary 3.1. Moreover, since $\chi_{\Sigma>1}(G)=1$ if and only if $G$ is a graph such that the degrees of every two adjacent vertices differ by at least 2 , we get $\chi_{\Sigma>1}\left(P_{\ell}\right) \geq 2$ for every $\ell \geq 2$.

Let $v_{0}, \ldots, v_{\ell}$ denote the vertices of the path $P_{\ell}$, with $v_{i} v_{i+1}$ being an edge for every $i$, $0 \leq i \leq \ell-1$. We clearly have $\chi_{\Sigma>1}\left(P_{2}\right)=2$ since the weighting $\omega$ given by $\omega\left(v_{0} v_{1}\right)=$ $\omega\left(v_{1} v_{2}\right)=2$ is neighbour-sum-2-distinguishing. Suppose now that $\ell \geq 3$. Then $P_{\ell}$ has two adjacent vertices $v_{i}$ and $v_{i+1}$ with degree 2, and Observation 3.3 applies. For any 2-edge-weighting $\omega$ of $P_{\ell}$, we have $\sigma\left(v_{i}\right)=\omega\left(v_{i-1} v_{i}\right)+\omega\left(v_{i} v_{i+1}\right)$ and $\sigma\left(v_{i+1}\right)=\omega\left(v_{i} v_{i+1}\right)+$ $\omega\left(v_{i+1} v_{i+2}\right)$. Since $\omega\left(v_{i-1} v_{i}\right), \omega\left(v_{i+1} v_{i+2}\right) \in\{1,2\}$, necessarily $\sigma\left(v_{i}\right)$ and $\sigma\left(v_{i+1}\right)$ differ by at most 1 , so that $\omega$ cannot be neighbour-sum-2-distinguishing.

If $\ell=3$, then assigning successive edge weights $1,3,3$ to the edges of $P_{3}$ is neighboursum-2-distinguishing as it yields successive incident sums $1,4,6,3$. So we may suppose from now on that $\ell \geq 4$. Under that assumption, note that a neighbour-sum-2-distinguishing 3 -edge-weighting $\omega$ of $P_{\ell}$ cannot assign weight 2 since Observation 3.3 applies. We thus restrict our attention to $\{1,3\}$-edge-weightings of $P_{\ell}$. Note that the edge $v_{1} v_{2}$ (and similarly $\left.v_{\ell-1} v_{\ell}\right)$ must be assigned weight 3 so that $\sigma\left(v_{0}\right)$ and $\sigma\left(v_{1}\right)$ differ by at least 2 . From this, observe that all neighbour-sum-2-distinguishing $\{1,3\}$-edge-weightings of $P_{\ell}$ are (up to directional symmetry) $1,3,3,1,1,3,3, \ldots, 3,3,1$, and $1,3,3,1,1, \ldots, 3,3$, and $3,3,1,1,3,3,1,1, \ldots, 3,3$. The claim then follows from the fact that these edge-weightings only apply for particular values of $\ell$ modulo 4 . 


\subsection{Cycles}

For every $\ell \geq 3$, we denote by $C_{\ell}$ the cycle of length $\ell$. In the next result, we determine the value of $\chi_{\Sigma>1}\left(C_{\ell}\right)$ for every cycle $C_{\ell}$.

Theorem 3.5. For every cycle $C_{\ell}, \ell \geq 3$, we have

$$
\chi_{\Sigma>1}\left(C_{\ell}\right)= \begin{cases}3 & \text { if } \ell \equiv 0 \quad(\bmod 4) \\ 5 & \text { otherwise. }\end{cases}
$$

Proof. Observe first that since cycles satisfy the 1-2-3 Conjecture and are regular, Corollary 2.4 implies that $\chi_{\Sigma>1}\left(C_{\ell}\right) \leq 5$ holds for every cycle $C_{\ell}$.

Let $v_{0}, \ldots, v_{\ell-1}$ denote the vertices of the cycle $C_{\ell}$, with $v_{i} v_{i+1}$ being an edge for every $i$, $0 \leq i \leq \ell-1$ (here and in the following, all operations over the subscripts are understood modulo $\ell$ ). Note first that Observation 3.3 implies that no cycle $C_{\ell}$ verifies $\chi_{\Sigma>1}\left(C_{\ell}\right) \leq 2$. Therefore, $\chi_{\Sigma>1}\left(C_{\ell}\right) \geq 3$ for every cycle $C_{\ell}$. Still according to Observation 3.3, note that no neighbour-sum-2-distinguishing 3-edge-weighting $\omega$ of $C_{\ell}$ can use weight 2 , Therefore, a neighbour-sum-2-distinguishing 3-edge-weighting of $C_{\ell}$ can only use weights 1 and 3 . In such a weighting, the edge weights must follow the pattern $1,1,3,3,1,1,3,3, \ldots$ along the cycle, which is possible if and only if $\ell \equiv 0(\bmod 4)$.

We now prove that $\chi_{\Sigma>1}\left(C_{\ell}\right)=5$ whenever $\ell \not \equiv 0(\bmod 4)$. Assume there is a neighbour-sum-2-distinguishing 4-edge-weighting $\omega$ of some cycle $C_{\ell}$. Note that, by Observation 3.3, any two edges $u v$ and $v w$ being at distance 2 must be assigned a small ( 1 or 2 ) and a big (3 or 4$)$ weight by $\omega$. Obviously, this cannot be completely achieved when $\ell \neq \equiv 0$ $(\bmod 4)$, while it is the case otherwise (for instance, follow the pattern $1,1,4,4,1,1,4,4, \ldots$ along $\left.C_{\ell}\right)$. This contradicts the existence of $\omega$, and, thus, $\chi_{\Sigma>1}\left(C_{\ell}\right)=5$ whenever $\ell \not \equiv 0$ $(\bmod 4)$.

\subsection{Odd multicacti}

Recall that $\chi_{\Sigma>1}(G) \leq 5$ holds for every odd multicactus $G$, according to Theorem 3.2. In the next result, we prove that, for odd multicacti, we can even design neighbour-sum2-distinguishing 5-edge-weightings that do not use weights 2 and 4. This supports Conjecture 5.1 we raise in the concluding section.

Observe first that connected multicacti can be defined inductively, as follows. Cycles of length at least 6 and congruent to 2 modulo 4, with edges coloured green and red alternatively, are multicacti. Consider now a multicactus $G$ whose edges are coloured green and red, and let $u v$ be a green edge of $G$. Then the graph obtained from $G$ by identifying $u$ and $v$ with the end-vertices of a path of length at least 5 and congruent to 1 modulo 4, whose edges are alternatively coloured red, green, ..., red (from one end to the other), is a multicactus. This operation will be referred to as a path attachment. Note that, in any edge-coloured multicactus, the two ends of a green edge have the same degree.

Theorem 3.6. Every odd multicactus admits a neighbour-sum-2-distinguishing $\{1,3,5\}$ edge-weighting.

Proof. Let $G$ be an odd multicactus. The proof is by induction on the number of path attachments performed to construct $G$. If no such path attachment was made, then $G$ is a cycle $C_{4 k+2}$, for some $k \geq 1$, and the $\{1,3,5\}$-edge-weighting obtained by applying the pattern $1,1,3,3,5,5,1,1,3,3,5,5, \ldots$ cyclically is clearly a neighbour-sum-2-distinguishing $\{1,3,5\}$-edge-weighting of $G$.

Assume now that $G$ is not a cycle. Then $G$ must contain a green edge $u v$ such that $u$ and $v$ are joined by exactly $x$ paths $P_{1}, \ldots, P_{x}, x \geq 1$, with length at least 5 and congruent 
to 1 modulo 4 , and whose internal vertices have degree 2 in $G$. In other words, no green edge of the $P_{i}$ 's was used to make a path attachment. In the extremal case where the $P_{i}$ 's cover all vertices of $G$ (i.e., all path attachments were performed on only one green edge), we omit one of these paths. Then, when removing all internal vertices of the $P_{i}$ 's from $G$, we get another connected odd multicactus $G^{\prime}$ in which both $u$ and $v$ have degree 2 . In particular, $G^{\prime}$ cannot be reduced to a single edge.

By the induction hypothesis, $G^{\prime}$ admits a neighbour-sum-2-distinguishing $\{1,3,5\}$-edgeweighting $\omega$, which we would like to extend to the edges of the $P_{i}$ 's, in order to obtain a neighbour-sum-2-distinguishing $\{1,3,5\}$-edge-weighting of $G$. Let us denote by $u^{\prime}$ and $v^{\prime}$ the neighbours of $u$ and $v$, respectively, different from $v$ and $u$, respectively, in $G^{\prime}$. When extending $\omega$ to the $P_{i}$ 's, we have to make sure that:

1. $\sigma(u)$ and $\sigma\left(u^{\prime}\right)$ still differ by at least 2 ;

2. $\sigma(v)$ and $\sigma\left(v^{\prime}\right)$ still differ by at least 2 ;

3. both $\sigma(u)$ and $\sigma(v)$ differ by at least 2 from the incident sums of their $x$ neighbours in the $P_{i}$ 's;

4. $\sigma(u)$ and $\sigma(v)$ still differ by at least 2 .

In order to respect the fourth condition above, we will edge-weight the $P_{i}$ 's in such a way that $\sigma(u)$ and $\sigma(v)$ are altered the same way, i.e., by a same integer $\alpha$. To that aim, we will $\{1,3,5\}$-edge-weight every $P_{i}$ in such a way that its two end-edges are assigned the same weight $\alpha$. In what follows, the main weighting scheme we use to extend $\omega$ to the consecutive edges of some $P_{i}$ is $\alpha, \beta, \beta, \gamma, \gamma, \beta, \beta, \ldots, \beta, \beta, \gamma, \alpha$ (or reversely), where $\alpha, \beta, \gamma \in\{1,3,5\}$ and $\alpha \neq \beta$ and $\beta \neq \gamma$. Note that, by that edge-weighting, for any such $\alpha, \beta, \gamma$ no two adjacent inner vertices have their sums differing by strictly less than 2 (recall Observation 3.3).

We consider the worst-case scenario where the number $x$ of $P_{i}$ 's is exactly 1 . This is indeed the worst case as, as $x$ gets larger, we get more and more ways to weight the $P_{i}$ 's, and thus more and more ways to extend $\omega$ correctly to $G$.

If $\omega(u v)=1$, then we set $\omega(u v)=3$. If no conflict arises, then we proceed with the next step. Otherwise, we proceed as follows. If there exists $\alpha \in\{1,3,5\}$ such that $\sigma(u)+\alpha$ and $\sigma\left(u^{\prime}\right)$ differ by at least 2, and similarly for $\sigma(v)+\alpha$ and $\sigma\left(v^{\prime}\right)$, then we extend $\omega$ as follows. Note first that, because $\omega(u v)=3$, we have, say, $\sigma(u) \geq 4$ and $\sigma(v) \geq 6(\sigma(u)<\sigma(v))$ by the induction hypothesis. We then extend $\omega$ to $P_{1}$ in the following way; in any case, it can be checked that the resulting edge-weighting of $G$ is correct:

- If $\alpha=1$, then we assign weights $1,3,3,1,1,3,3, \ldots, 3,3,1,1$ (i.e., $\alpha=1, \beta=3$, $\gamma=1$ ) to the edges of $P_{1}$ as going from $v$ to $u$.

- If $\alpha=3$, then we assign weights $3,1,1,3,3,1,1, \ldots, 1,1,3,3$ (i.e., $\alpha=3, \beta=1$, $\gamma=3$ ) as going from $u$ to $v$.

- If $\alpha=5$, then we change $\omega(u v)$ from 3 to 5 and $\alpha$ from 5 to 3 , so that the previous case now applies.

Now, if no such $\alpha$ exists, then we set $\omega(u v)=5$. In the present situation, $\alpha=5$ now has the desired properties. Similar extension arguments then apply.

So now assume that $\omega(u v) \in\{3,5\}$ and there is currently no sum conflict. Again, if there exists $\alpha \in\{1,3,5\}$ with the desired properties, then we are done. Otherwise, 
note that no conflict may arise when setting $\omega(u v)=1$ (as otherwise there would exist an $\alpha$ when $\omega(u v) \in\{3,5\})$, and $\alpha=1$ is as required. Since $\omega(u v)=1$, we have, say, $2 \leq \sigma(u) \leq 4$ and $4 \leq \sigma(v) \leq 6(\sigma(u)<\sigma(v))$. We then extend $\omega$ to the edges of $P_{1}$ as follows:

- If $\sigma(u)=2$ and $\sigma(v) \in\{4,6\}$, then we assign weights $1,5,5,1,1,5,5, \ldots, 5,5,1,1$ (i.e., $\alpha=1, \beta=5, \gamma=1$ ) to the edges of $P_{1}$ as going from $u$ to $v$.

- If $\sigma(u)=4$ and $\sigma(v)=6$, then we assign weights $1,3,3,1,1,3,3, \ldots, 3,3,1,1$ (i.e., $\alpha=1, \beta=3, \gamma=1$ ) as going from $v$ to $u$.

In all cases, it can be checked that no sum conflict arises in $G$, and thus we get a neighboursum-2-distinguishing $\{1,3,5\}$-edge-weighting. This completes the proof.

\section{Algorithmic aspects}

In this section, we consider the hardness of determining the value of $\chi_{\Sigma>1}(G)$ for a given graph $G$. We first prove, in Subsection 4.1, that deciding whether $\chi_{\Sigma>1}(G) \leq 2$ holds for a given graph $G$ is NP-complete, even when restricted to 3-degenerate planar bipartite graphs. In Subsection 4.2, we prove that, although $\chi_{\Sigma>1}(T)$ can take any value in $\{1,2,3,4\}$ for a given tree $T$ (recall Corollary 3.1), deciding the exact value of $\chi_{\Sigma>1}(T)$ can be done in polynomial time.

It is worth recalling that determining the value of $\chi_{\Sigma}(G)$ for a given graph $G$ is NPcomplete in general (Dudek and Wajc [1]), but can be done in polynomial time when restricted to bipartite graphs (Thomassen, Wu and Yang [7]). Hence our result in Subsection 4.1 shows another difference between the parameters $\chi_{\Sigma}$ and $\chi_{\Sigma>1}$.

\subsection{General case}

Before proceeding with the proof of the main result of this subsection, we first introduce gadgets that we will use to force some weights to be used by any neighbour-sum-2distinguishing 2-edge-weighting. Each of these gadgets will have a root vertex of degree 1 being incident to a root edge. We here relax the notion of neighbour-sum-2-distinguishing 2-edge-weighting around the root; that is, we allow a neighbour-sum-2-distinguishing 2edge-weighting to have adjacent incident sums differing by less than 2 , but the incident sum of the root has to be involved in such a conflict. This is because our gadgets will be attached to other graphs via the root, so, in the properties we point out below, the incident sum of the root should not be regarded as fixed.

The gadgets we will construct are called $(\alpha, S)$-gadgets, for some given $\alpha \in\{1,2\}$ and $S \subset \mathbb{N}^{*}(S \neq \emptyset)$. Every such gadget $G$ will satisfy the two following properties:

1. the root edge of $G$ is necessarily weighted $\alpha$ by any neighbour-sum-2-distinguishing 2-edge-weighting of $G$, and

2. $S$ is the set of all numbers $s$ such that there exists a neighbour-sum-2-distinguishing 2-edge-weighting of $G$ where the (unique) neighbour of the root has incident sum $s$.

These gadgets will be used as follows. Let $H$ be a graph and $v$ be a vertex of $H$. Add to $H$ an $(\alpha, S)$-gadget $G$ (for some $\alpha$ and $S$ ), and identify $v$ with the root of $G$. Then, in any neighbour-sum-2-distinguishing 2-edge-weighting of $H, v$ will necessarily receive weight $\alpha$ from the root edge of $G$, and $v$ will be adjacent to a vertex whose incident sum belongs to $S$. This mechanism can be used both to force particular edge weights to appear around $v$, and to ensure that $\sigma(v)$ does not belong to a particular set (in particular when $|S|=1$ ). 

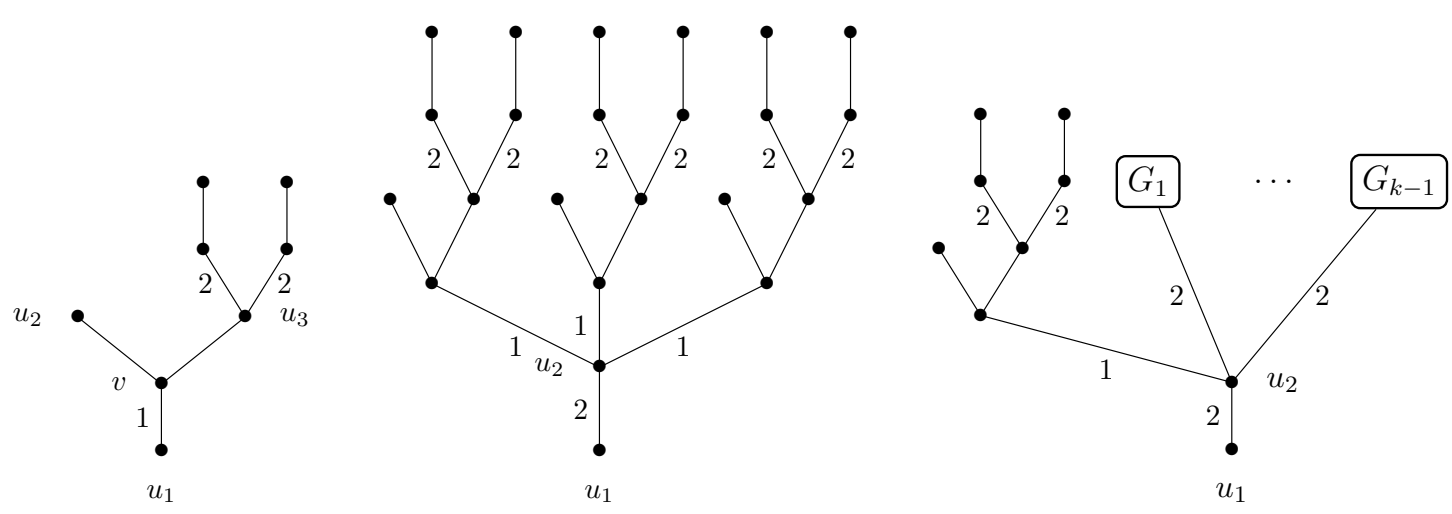

Figure 1: The $(1,\{3,4\})$-gadget with root $u_{1}$ (left), the $(2,\{5\})$-gadget with root $u_{1}$ (middle), and the $(2,\{2 k+1\})$-gadget with root $u_{1}$ (right).

We now introduce the gadgets we will use (see Figure 1 for an illustration). Consider first a path $G:=u_{1} u_{2} u_{3}$ of length 2 . We claim that $G$ is a $(2,\{3,4\})$-gadget with root $u_{1}$. Indeed, in any neighbour-sum-2-distinguishing 2-edge-weighting $\omega$ of $G$ (with the relaxation mentioned above), we have $\omega\left(u_{1} u_{2}\right)=2$, while $\omega\left(u_{2} u_{3}\right)$ can have value either 1 or 2 , in which cases we get $\sigma\left(u_{2}\right)=3$ and $\sigma\left(u_{2}\right)=4$, respectively.

Now consider a claw $G$ with vertices $v, u_{1}, u_{2}, u_{3}$, where the $u_{i}$ 's are the leaves. Add two $(2,\{3,4\})$-gadgets $G_{1}$ and $G_{2}$ to $G$, and identify $u_{3}$ and the roots of $G_{1}$ and $G_{2}$. We claim that $G$ is a $(1,\{3,4\})$-gadget with root $u_{1}$. In any neighbour-sum-2-distinguishing 2-edgeweighting $\omega$ of $G$, the vertex $u_{3}$ is incident to at least two edges with weight 2 (because of the gadgets $G_{1}$ and $G_{2}$ ), so that $\sigma\left(u_{3}\right)=5\left(\right.$ if $\omega\left(u_{3} v\right)=1$ ) or $\sigma\left(u_{3}\right)=6$ (otherwise). In both cases, we necessarily have $\omega\left(v u_{2}\right)=\omega\left(v u_{1}\right)=1$, so that $\sigma(v)$ and $\sigma\left(u_{3}\right)$ differ by at least 2. We thus get $\sigma(v)=3$ in the first case, and $\sigma(v)=4$ in the second case.

We now describe how to obtain $(2, S)$-gadgets with $S:=\{2 k+1\}$ for any $k \geq 2$. We first build a $(2,\{5\})$-gadget as follows. Start from $G:=u_{1} u_{2}$ being the path of length 1 , then add three $(1,\{3,4\})$-gadgets $G_{1}, G_{2}, G_{3}$ to $G$, and identify $u_{2}$ and the roots of $G_{1}$, $G_{2}$ and $G_{3}$. We claim that $G$ is a $(2,\{5\})$-gadget with root $u_{1}$. In any neighbour-sum2-distinguishing 2-edge-weighting $\omega$ of $G$, the vertex $u_{2}$ is incident to at least three edges weighted 1 , namely the root edges of the $G_{i}$ 's. Now, if $\omega\left(u_{1} u_{2}\right)=1$, then $\sigma\left(u_{2}\right)=4$, which creates sum conflicts with vertices from the $G_{i}$ 's. So we necessarily have $\omega\left(u_{1} u_{2}\right)=2$, in which case $\sigma\left(u_{2}\right)=5$, which is fine since the $G_{i}$ 's are $(1,\{3,4\})$-gadgets.

We now turn to the general case. Let $2 k+1 \geq 7$, and assume that we have constructed $\left(2, S^{\prime}\right)$-gadgets with $S^{\prime}:=\left\{2 k^{\prime}+1\right\}$ for every $k^{\prime}, 2 \leq k^{\prime}<k$. Start from $G:=u_{1} u_{2}$ being the path of length 1 . Add $k-1(2,\{2 k-1\})$-gadgets $G_{1}, \ldots, G_{k-1}$ to $G$, as well as one $(1,\{3,4\})$-gadget $G_{0}$, and identify $u_{2}$ and the roots of $G_{1}, \ldots, G_{k-1}$ and $G_{0}$. We claim that $G$ is a $(2,\{2 k+1\})$-gadget with root $u_{1}$. In any neighbour-sum-2-distinguishing 2-edgeweighting $\omega$ of $G$, the $G_{i}$ 's force $\sigma\left(u_{2}\right)$ to have value at least $2 k-1$. Depending on whether $\omega\left(u_{1} u_{2}\right)=1$ or $\omega\left(u_{1} u_{2}\right)=2$, we thus have $\sigma\left(u_{2}\right)=2 k$ or $\sigma\left(u_{2}\right)=2 k+1$, respectively. In the first case, we get sum conflicts between $u_{2}$ and its neighbours in $G_{1}, \ldots, G_{k-1}$ since their incident sums differ by 1 . Therefore, we necessarily have $\omega\left(u_{1} u_{2}\right)=2$, so that $\sigma\left(u_{2}\right)=2 k+1$, which produces no sum conflict in $G$.

Analogous $(1, S)$-gadgets with $S:=\{2 k+1\}, k \geq 2$, will also be needed. A $(1,\{5\})$ gadget can be obtained as follows. Start from $G:=u_{1} u_{2}$ being the path of length 1 , add two $(2,\{7\})$-gadgets $G_{1}$ and $G_{2}$ to $G$, and identify $u_{2}$ and the roots of $G_{1}$ and $G_{2}$. In any neighbour-sum-2-distinguishing 2-edge-weighting $\omega$ of $G$, the $G_{i}$ 's force $u_{2}$ to be incident to at least two edges with weight 2. So we have $\sigma\left(u_{2}\right)=5$ or $\sigma\left(u_{2}\right)=6$ depending on whether 
$\omega\left(u_{1} u_{2}\right)=1$ or $\omega\left(u_{1} u_{2}\right)=2$, respectively. In the second case, however, we get sum conflicts between $u_{2}$ and its neighbours in $G_{1}$ and $G_{2}$. We thus necessarily have $\omega\left(u_{1} u_{2}\right)=1$ and $\sigma\left(u_{2}\right)=5$, which produces no sum conflict in $G$.

Now let $2 k+1 \geq 7$, and assume that we have constructed $\left(1, S^{\prime}\right)$-gadgets with $S^{\prime}:=$ $\left\{2 k^{\prime}+1\right\}$ for every $k^{\prime}, 2 \leq k^{\prime}<k$. Start from $G:=u_{1} u_{2}$ being the path of length 1 , add $k(2,\{2 k+3\})$-gadgets $G_{1}, \ldots, G_{k}$ to $G$, and identify $u_{2}$ and the roots of $G_{1}, \ldots, G_{k}$. We claim that $G$ is a $(1,\{2 k+1\})$-gadget. In any neighbour-sum-2-distinguishing 2-edgeweighting $\omega$ of $G$, the $G_{i}$ 's force $\sigma\left(u_{2}\right)$ to be at least $2 k$. Depending on whether $\omega\left(u_{1} u_{2}\right)=1$ or $\omega\left(u_{1} u_{2}\right)=2$, we thus have $\sigma\left(u_{2}\right)=2 k+1$ or $\sigma\left(u_{2}\right)=2 k+2$, respectively. In the second case, we get sum conflicts between $u_{2}$ and its neighbours in $G_{1}, \ldots, G_{k}$, since their incident sums differ by 1 . Therefore, we necessarily have $\omega\left(u_{1} u_{2}\right)=1$ and $\sigma\left(u_{2}\right)=2 k+1$, which produces no sum conflict in $G$.

Note that all the above-constructed gadgets are trees. With all these gadgets in hand, we now prove the main result of this section.

Theorem 4.1. For a given 3 -degenerate planar bipartite graph $G$, deciding whether $\chi_{\Sigma>1}(G) \leq$ 2 holds is NP-complete.

Proof. Since the problem is obviously in NP, we proceed with the proof of its NP-hardness. The proof is by reduction from 1-IN-3 SAT. From a formula $F$, we construct a graph $G$ such that $F$ is satisfiable in a 1-in-3 way if and only if $G$ admits a neighbour-sum-2distinguishing 2-edge-weighting. Since the Monotone version of 1-IN-3 SAT remains NP-complete (see e.g. [5]), we may assume that $F$ has no negated variables. Also, we may assume that all clauses of $F$ have three distinct variables, as otherwise $F$ could be simplified. That is:

- if $F$ has a clause $\left(x_{i_{1}} \vee x_{i_{1}} \vee x_{i_{1}}\right)$, then $F$ is not satisfiable in a 1-in-3 way;

- if $F$ has a clause $\left(x_{i_{1}} \vee x_{i_{1}} \vee x_{i_{2}}\right)$, then $x_{i_{2}}$ and $x_{i_{1}}$ are forced to true and false, respectively, by any truth assignment making $F$ satisfied in a 1-in-3 way.

We denote by $x_{1}, \ldots, x_{n}$ the variables of $F$, and by $C_{1}, \ldots, C_{m}$ its clauses. The construction of $G$, which is clearly achieved in polynomial time, is as follows. We start by adding variable gadgets in the following way. For each variable $x_{i}$ of $F$, we add to $G$ a star $V_{i}$ with root $v_{i}$ and $2 k_{i}$ leaves $u_{i, 1}, \ldots, u_{i, 2 k_{i}}$, where $2 k_{i} \geq \max \left\{10, n_{i}\right\}$ is any even integer, and $n_{i}$ is the number of clauses of $F$ that contain $x_{i}$. Next we add $\left(1,\left\{4 k_{i}+1\right\}\right)$-, $\left(2,\left\{4 k_{i}+3\right\}\right)-,\left(2,\left\{4 k_{i}+5\right\}\right)-, \ldots,\left(2,\left\{6 k_{i}-3\right\}\right)$ - and $\left(2,\left\{6 k_{i}-3\right\}\right)$-gadgets $G_{1}, \ldots, G_{k_{i}}$ to $G$, and identify $v_{i}$ and the roots of $G_{1}, \ldots, G_{k_{i}}$. To avoid any ambiguity, let us emphasize that, for instance, when $k_{i}=5$, the five added gadgets are one $(1,\{21\})$-gadget, one $(2,\{23\})$-gadget, one $(2,\{25\})$-gadget and two $(2,\{27\})$-gadgets.

Because of the $G_{i}$ 's, in any neighbour-sum-2-distinguishing 2-edge-weighting $\omega$ of $V_{i}$, the value of $\sigma\left(v_{i}\right)$ lies between $4 k_{i}-1$ (when all $v_{i} u_{i, j}$ 's are assigned weight 1 ) and $6 k_{i}-1$ (when all $v_{i} u_{i, j}$ 's are assigned weight 2). Furthermore, $4 k_{i}-1$ and $6 k_{i}-1$ are both odd. Moreover, we cannot have $\sigma\left(v_{i}\right) \in\left\{4 k_{i}, \ldots, 6 k_{i}-2\right\}$ as otherwise there would be a sum conflict involving $v_{i}$ and one of its neighbours in the $G_{i}$ 's. Therefore, either all $\omega\left(v_{i} u_{i, j}\right)$ 's are equal to 1 , or all $\omega\left(v_{i} u_{i, j}\right)$ 's are equal to 2 . In what follows, we call the vertices $u_{i, 1}, \ldots, u_{i, 2 k_{i}}$ the output vertices of $V_{i}$, and the edges $v_{i} u_{i, 1}, \ldots, v_{i} u_{i, 2 k_{i}}$ the output edges of $V_{i}$,

We now modify $G$ by considering the clauses of $F$. For each clause $C_{j}=\left(x_{i_{1}} \vee x_{i_{2}} \vee x_{i_{3}}\right)$ of $F$, we add a clause vertex $c_{j}$ to $G$. For each $V_{i_{1}}, V_{i_{2}}, V_{i_{3}}$, we then select one output vertex still having degree 1 , and identify $c_{j}$ and the three selected output vertices. Finally, we add 
a $(2,\{7\})$-gadget $G_{1}$ to $G$, as well as a $(2,\{11\})$-gadget $G_{2}$, and identify $c_{j}$ and the roots of $G_{1}$ and $G_{2}$. In any neighbour-sum-2-distinguishing 2-edge-weighting $\omega$ of $G, \sigma\left(c_{j}\right)$ has thus value at least 4 (because of $G_{1}$ and $G_{2}$ ), and ranges in $\{7, \ldots, 10\}$. However, $\sigma\left(c_{j}\right)$ cannot take any value among $\{7,8,10\}$ because of $G_{1}$ and $G_{2}$. So we necessarily have $\sigma\left(c_{j}\right)=9$, which occurs only if exactly one of the three output edges originating from $V_{i_{1}}, V_{i_{2}}, V_{i_{3}}$ is assigned weight 1 .

It can be checked that no unexpected sum conflicts (that is, different from those listed above) can arise, in particular thanks to our choice of the $2 k_{i}$ 's. We now claim that we have the desired equivalence. This directly follows from the following arguments:

- Assigning weight 1 (resp. 2) to an output edge $v_{i} c_{j}$ simulates the fact that variable $x_{i}$ brings truth value true (resp. false) to $C_{j}$.

- Following that equivalence, the fact that, for any $V_{i}$, all output edges of $V_{i}$ must be weighted 1 (resp. 2) simulates the fact that setting $x_{i}$ to true (resp. false) by some truth assignment brings the same truth value to every clause containing $x_{i}$.

- The fact that, for every clause vertex $c_{j}$, exactly one incident output edge must be assigned weight 1 simulates the fact that a clause of $F$ is considered satisfied if and only if it includes exactly one true variable.

To complete the proof of the theorem, it suffices to observe the following:

- The Planar version of Monotone 1-IN-3 SAT remains NP-complete (see [5]), so we may assume that $F$ is a planar formula. Since every gadget is a tree, the construction above then yields a planar $G$.

- Since every gadget is a tree, the graph $G$ is 3 -degenerate.

- The only cycles in $G$ are those of the subgraph induced by the $v_{i}$ 's and the $c_{j}$ 's. Since this subgraph is bipartite, so is $G$.

This concludes the proof.

\subsection{Tree case}

In this section, we prove that the counterpart of Theorem 4.1 for trees is not true. That is, we prove that determining the value of $\chi_{\Sigma>1}(T)$ for a given tree $T$ can be done in polynomial time. Recall that for a tree $T$, we always have $\chi_{\Sigma>1}(T) \leq 4$ (according to Corollary 3.1), while $\chi_{\Sigma>1}(T)=1$ if and only if, for every two adjacent vertices $u$ and $v$ of $T$, the values of $d(u)$ and $d(v)$ differ by at least 2 .

Theorem 4.2. For a given tree $T$, determining $\chi_{\Sigma>1}(T)$ can be done in polynomial time.

Proof. For any fixed $k \in\{1,2,3,4\}$, we introduce below an algorithm that checks in polynomial time whether $T$ admits a neighbour-sum-2-distinguishing $k$-edge-weighting. So, to determine $\chi_{\Sigma>1}(T)$, we can essentially run this algorithm successively with $k=1,2,3,4$. The first value of $k$ for which the algorithm answers positively is the value of $\chi_{\Sigma>1}(T)$.

Designate a node $r$ of $T$ as being its root. This defines a root-to-leaf orientation of $T$ in the usual way, where every non-root node $v$ has a parent, and every non-leaf node $v$ has children. By the descendants of $v$, we refer to the nodes of $T$ for which we find $v$ when iterating the parent relationship. 
The subtree $T_{v}$ of $T$ rooted at $v$ is the subtree whose nodes are $v$ and all its descendants. This subtree $T_{v}$ can itself be decomposed into several subtrees, in the following way. Assume that $v$ has $d \geq 1$ descendants $u_{1}, \ldots, u_{d}$, ordered following an arbitrary order (supposed to be fixed throughout the proof). Then $T_{v}$ can be edge-decomposed into $d$ subtrees $T_{v, 1}, \ldots, T_{v, d}$ being $T_{u_{1}}+v u_{1}, \ldots, T_{u_{d}}+v u_{d}$, respectively, whose root, $v$, has degree precisely 1 . Trees with this property are called shrubs throughout. Conversely, $T_{v}$ is obtained by identifying the roots of the shrubs $T_{v, 1}, \ldots, T_{v, d}$. For every shrub, we call the edge incident to the root the root edge. The non-root end of the root edge is called the subroot.

We are now ready to describe our algorithm for deciding whether $T$ admits a neighboursum-2-distinguishing $k$-edge-weighting. The rough ideas are the following. The tree $T$ can be seen as a union of $d:=d(r)$ shrubs $S_{1}, \ldots, S_{d}$ whose roots were identified, resulting in $r$. A neighbour-sum-2-distinguishing $k$-edge-weighting of $T$ is thus essentially the union of (relaxed, see below) neighbour-sum-2-distinguishing $k$-edge-weightings of the $d$ shrubs attached to $r$, with the additional property that the resulting $\sigma(r)$ does not create any sum conflict. Therefore, in order to construct a neighbour-sum-2-distinguishing $k$-edge-weighting of $T$, it suffices to find convenient neighbour-sum-2-distinguishing $k$-edgeweightings of $S_{1}, \ldots, S_{d}$ that can be "glued". So we need to know, for each shrub $S_{i}$ and for every $\alpha \in\{1, \ldots, k\}$, whether $S_{i}$ admits a neighbour-sum-2-distinguishing $k$-edgeweighting where the root edge is assigned colour $\alpha$, and, for such an edge-weighting of $S_{i}$, which possible incident sums can be obtained for the subroot.

More formally, for a shrub $S$ with root $v^{\prime}$ and subroot $v$, we want to compute, for every weight $\alpha \in\{1, \ldots, k\}$, the set $X_{\alpha}(v)$ of possible values of $\sigma(v)$ by a neighbour-sum-2distinguishing $k$-edge-weighting of $S$ assigning weight $\alpha$ to $v^{\prime} v$. Note that a shrub might be a single edge, and may thus admit no neighbour-sum-2-distinguishing $k$-edge-weighting. In that special case, we relax the notion of neighbour-sum-2-distinguishing $k$-edge-weighting, and allow the root and the subroot to have the same incident sums.

Assume $v$ has $d$ children $u_{1}, \ldots, u_{d}, d \geq 0$, and let $S_{1}, \ldots, S_{d}$ denote the $d$ shrubs attached to $v$ in $S$. We claim that each $X_{\alpha}(v)$ can be computed in polynomial time from the sets

$$
X_{1}\left(u_{1}\right), \ldots, X_{k}\left(u_{1}\right), X_{1}\left(u_{2}\right), \ldots, X_{k}\left(u_{2}\right), \ldots, X_{1}\left(u_{d}\right), \ldots, X_{k}\left(u_{d}\right),
$$

computed by induction for the shrubs $S_{1}, \ldots, S_{d}$. So, in a way, the sets $X_{1}(v), \ldots, X_{k}(v)$ can be computed from smaller shrubs, and deduced successively towards the subroot of $S$. We prove this below.

The base case is when $S$ is a single edge, that is, $v$ has no child. If the edge $v^{\prime} v$ is assigned any weight $\alpha \in\{1, \ldots, k\}$ by a neighbour-sum-2-distinguishing $k$-edge-weighting, then $\sigma(v)=\alpha$. So, for such a shrub $S$, we have $X_{\alpha}(v)=\{\alpha\}$ for every $\alpha \in\{1, \ldots, k\}$.

Suppose now that $v$ has $d \geq 1$ children $u_{1}, \ldots, u_{d}$, and, for each shrub $S_{i}, 1 \leq i \leq d$, attached to $v$, and every $\alpha \in\{1, \ldots, k\}$, the set $X_{\alpha}\left(u_{i}\right)$ has been computed by induction. We now want to compute the sets $X_{1}(v), \ldots, X_{k}(v)$. Since $d(v)=d+1$, by any $k$-edgeweighting of $S$, the sum $\sigma(v)$ can take up to $k d+k-d$ values, namely those among $\{d+1, \ldots, k d+k\}$. We repeatedly fix one of those sums $x$, and we determine whether $x$ can be added to some of the sets $X_{1}(v), \ldots, X_{k}(v)$.

Assume we want to determine whether $x$ has to be added to $X_{\alpha}(v)$, where $\alpha$ is any value in $\{1, \ldots, k\}$. Successively consider all partitions $x_{1}+2 x_{2}+\cdots+k x_{k}$ of $x$ into $x_{1}+\cdots+x_{k}=d+1$ values among $\{1, \ldots, k\}$ only. Recall that we are focusing on computing $X_{\alpha}(v)$, so if $x_{\alpha}=0$, then we can consider the next partition of $x$. Since $x$ is linear in $|V(T)|$ and $k \leq 4$ is fixed, the number of such partitions to consider is polynomial in $|V(T)|$. Essentially, we have $x_{1}, \ldots, x_{k} \leq|V(T)|$, meaning that the number of such 
partitions is roughly $|V(T)|^{k-1}$. We now want to know if there is a neighbour-sum-2distinguishing $k$-edge-weighting of $S$ where $x_{i}$ edges incident to $v$ are assigned weight $i$, for every $i \in\{1, \ldots, k\}$. If such an edge-weighting exists, then for any $S_{i}$ to which the weight $\beta$ is assigned to the root edge, $X_{\beta}\left(u_{i}\right)$ contains some value not in $\{\alpha-1, \alpha, \alpha+1\}$.

Since we are focusing on $X_{\alpha}(v)$, one of the $x_{\alpha}$ weights $\alpha$ around $v$ will be assigned to $v^{\prime} v$. This leaves us with $d$ other weights to assign bijectively to the $v u_{i}$ 's, with the constraint that if we assign a weight $\beta$ to $v u_{i}$, then $X_{\beta}\left(u_{i}\right)$ should contain a value not among $\{\alpha-1, \alpha, \alpha+1\}$. If $\beta$ can indeed by assigned to $v u_{i}$ safely, then we call this a valid assignment. To find a correct assignation (if any exists), we build a compatibility bipartite graph $C$ of the valid assignments, as follows. In one side of the bipartition of $C$, we put $d$ vertices corresponding to the $d$ weights we want to assign. In the other side, we put $d$ vertices corresponding to the edges $v u_{1}, \ldots, v u_{d}$ of $S$. We then add an edge joining two vertices of $C$ if assigning the corresponding weight to the corresponding edge of $S$ is valid. Now, finding a satisfying assignment of the $d$ weights to the root edges of the $S_{i}$ 's is equivalent to finding a perfect matching in $C$, which is known to be doable in polynomial time. If there indeed exists such a perfect matching of $C$, then we add $x$ to $X_{\alpha}(v)$.

We now go back to $T$, with the root $r$ having $d$ children $u_{1}, \ldots, u_{d}$. For each $S_{i}$ of the $d$ shrubs $S_{1}, \ldots, S_{d}$ rooted at $v$, we can compute the sets $X_{1}\left(u_{i}\right), \ldots, X_{k}\left(u_{i}\right)$ as explained above. These sets memorize, in a compact way, all possible ways, in terms of incident sums and weights assigned to the root edges, to $k$-edge-weight the $S_{i}$ 's in a neighboursum-2-distinguishing way. Now, again, we can consider every potential incident sum $x$ as $\sigma(r)$, every potential way to partition $x$ into $d$ integers among $\{1, \ldots, k\}$, and, building the compatibility bipartite graph as above, find, if it exists, a valid way to bijectively assign the $d$ weights to the $d$ root edges $v u_{1}, \ldots, v u_{d}$. If a valid assignment for a partition of some $x$ exists, then $T$ admits a neighbour-sum-2-distinguishing $k$-edge-weighting. Otherwise, it does not.

Concerning the complexity aspect, determining $X_{\alpha}(v)$ for a shrub of $T$ with subroot $v$ can be done in polynomial time. Recall that $k \leq 4$ is constant. The number of possible sums $x$ as $\sigma(v)$ to consider is at most $k|V(T)|$. For each of these values of $x$, we consider up to $|V(T)|^{k-1}$ partitions into 1's, 2's, ..., and $k$ 's. Deciding whether there is a valid assignment for one of those partitions can be done in polynomial time, using for instance Edmonds' Blossom Algorithm for computing maximum matchings [2]. The procedure above is almost the same when $r$ is considered. By all these arguments, the whole procedure can be achieved in polynomial time.

\section{Conclusion}

In this work, we have investigated the consequences on the 1-2-3 Conjecture of requiring adjacent vertices to be distinguishable in a stronger way, namely by asking their incident sums to differ by at least 2 . We have addressed Conjecture 1.1, to which we did not manage to come up with any counterexample, as an equivalent of the 1-2-3 Conjecture in this context. As a main evidence that our conjecture might be true, we have pointed out some connections between the 1-2-3 Conjecture and Conjecture 1.1, and proved the later one for nice bipartite graphs.

Several aspects related to Conjecture 1.1 remain unclear to us, and could thus be subject to further work. First, we do not fully understand how necessary the weights 2 and 4 are for our conjecture. In particular, most graphs for which we have proved Conjecture 1.1 actually admit neighbour-sum-2-distinguishing $\{1,3,5\}$-edge-weightings (recall, in particular, Theorem 3.6). This supports the following refinement of Conjecture 1.1. 
Conjecture 5.1. Every nice graph admits a neighbour-sum-2-distinguishing $\{1,3,5\}$-edgeweighting.

In the context of neighbour-sum-2-distinguishing edge-weightings, Conjecture $5.1 \mathrm{might}$ actually be an equivalent to the 1-2-3 Conjecture more natural than Conjecture 1.1. Indeed, in the 1-2-3 Conjecture we aim at getting incident sums differing by at least 1 by using three successive weights $\alpha-1, \alpha, \alpha+1$ differing by 1 . In Conjecture 5.1, we aim at getting incident sums differing by at least 2 by using three "successive" weights $\alpha-2, \alpha, \alpha+2$ differing by 2 . Following this reasoning, perhaps, in general, the following conjecture might be the right direction to consider.

Conjecture 5.2. Let $d \geq 1$ be an integer. Every nice graph admits a neighbour-sum-ddistinguishing $\{1, d+1,2 d+1\}$-edge-weighting.

There are intriguing examples, though, such as nice paths $P_{\ell}$ of length congruent to 1 modulo 4 (for which $\chi_{\Sigma>1}\left(P_{\ell}\right)=4$, recall Theorem 3.4), showing that, for neighboursum-2-distinguishing edge-weightings, the weights 2 and 4 are sometimes worth using to get an optimal edge-weighting. More generally, our NP-hardness reduction in the proof of Theorem 4.1 shows that there exist complex examples for which an optimal edge-weighting uses weights 1 and 2 only. We believe this could be an interesting aspect to study further.

Although we have proved Conjecture 1.1 for nice bipartite graphs (Theorem 3.2), we have not proved refined Conjecture 5.1 for all such graphs. Another interesting line of research could thus be to generalize Theorem 3.6 to all nice bipartite graphs, which would be a first step towards Conjecture 5.1.

More directions for future works on neighbour-sum-2-distinguishing edge-weightings are also worth mentioning. Notably, we did not manage to improve the bounds given in Section 2 for many classes of graphs. Generally speaking, it does not seem obvious to us how to improve the bound in Corollary 2.2, and this would surely require new dedicated tools. Concerning particular classes of graphs, let us mention the case of subcubic graphs. Although we know that cubic graphs comply with Conjecture 1.1, and even Conjecture 5.1 (recall Corollary 2.4), we did not manage to prove that nice subcubic graphs, in general, also do. We believe this would be an appealing first case to consider towards proving Conjecture 1.1 for 3-chromatic graphs, for which the 1-2-3 Conjecture holds.

More generally, it would be interesting to consider Conjecture 5.2 above. Many of the arguments and techniques used in this work actually generalize to neighbour-sumdistinguishing $d$-edge-weightings. For values of $d$ larger than 2, it is likely that more intriguing phenomenon arise.

Acknowledgements. The authors are grateful to two anonymous referees for suggesting interesting additional results and proof simplifications, which appear in the current paper.

\section{References}

[1] A. Dudek, D. Wajc. On the complexity of vertex-coloring edge-weightings. Discrete Mathematics Theoretical Computer Science, 13(3):45-50, 2011.

[2] J. Edmonds. Paths, trees, and flowers. Canadian Journal of Mathematics, 17:449-467, 1965.

[3] M. Kalkowski, M. Karoński, F. Pfender. Vertex-coloring edge-weightings: towards the 1-2-3 Conjecture. Journal of Combinatorial Theory, Series B, 100:347-349, 2010. 
[4] M. Karoński, T. Łuczak, A. Thomason. Edge weights and vertex colours. Journal of Combinatorial Theory, Series B, 91:151-157, 2004.

[5] W. Mulzer, G. Rote. Minimum-weight triangulation is NP-hard. Journal of the ACM, 55(2), Article no. 11, 2008.

[6] B. Seamone. The 1-2-3 Conjecture and related problems: a survey. Technical report, available online at http://arxiv.org/abs/1211.5122, 2012.

[7] C. Thomassen, Y. Wu, C.-Q. Zhang. The 3-flow conjecture, factors modulo $k$, and the 1-2-3-conjecture. Journal of Combinatorial Theory, Series B, 121:308-325, 2016. 